\title{
LA DOMESTICACIÓN COMO ENTRECRUZAMIENTO ENTRE LOS MUNDOS HUMANO Y ANIMAL. UNA PERSPECTIVA FENOMENOLÓGICA Y NATURALISTA
}

\author{
Domestication As A Criss-cross Between The Human And The Animal's World. A \\ Phenomenological And Naturalist Perspective \\ Cristián Martínez Bravo \\ Universidad Diego Portales/Universidad de Santiago, Santiago, Chile \\ cristianmb1984@gmail.com
}

\begin{abstract}
Resumen
La domesticación puede ser entendida como un entrecruce entre seres humanos y animales. Fenomenológicamente hablando, esta corresponde a un caso en el cual los animales adquieren ciertos rasgos humanos que surgen a partir de la convivencia que se da entre ellos y los seres humanos. En la ciencia encontramos casos de orangutanes, tales como el de Chantek y Puti, los cuales adquirieron ciertas capacidades de lenguaje no verbal a partir de su exposición en diversos grados a contextos humanos. No obstante, tales capacidades dependían de la fuerza y la riqueza de tal convivencia. En este trabajo quiero mostrar el modo en que la domesticación, entendida como este entrecruzamiento entre la vida humana y animal, puede tener consecuencias ontológicas y éticas para la constitución de las subjetividades animales y humanas dependiendo de la fuerza y la riqueza de su convivencia.
\end{abstract}

Palabras clave: domesticación, entrecruzamiento, convivencia, constitución, apuntar.

\begin{abstract}
Domestication is understood as a criss-cross between humans and animals. Phenomenologically speaking, this phenomenon is a case in which animals acquire certain human traits that arise from the mutual coexistence between animals and human beings. Science has shown a case of two orangutans, namely, Chantek and Puti, who acquired certain non-verbal language capacities from the exposition in different degrees to human contexts. Nonetheless, such capacities depended on the force and richness of the coexistence between animals and human beings. In this paper, I want to show how domestication, understood as a criss-cross between human and animal life, could have ontological and ethical consequences for the constitution of animal and human subjectivities depending on the force and richness of the mutual coexistence between both of them.
\end{abstract}

Keywords: domestication, criss-cross, coexistence, constitution, pointing.

Fecha de Recepción: 05/11/2019 - Fecha de Aceptación: 26/12/2019 


\section{Introducción}

En la descripción del mundo de la vida en Crisis §55, Husserl señala que, en el sentido intersubjetivamente más pleno, este es un mundo de la cultura (Husserl 2008a 228). Con esta afirmación, Husserl quiere decir que el mundo de la vida es el mundo cotidiano donde habita el ser humano con sus intereses prácticos y teóricos, y que esencialmente es un mundo de evidencias subjetivo-relativas (2008a 168). Sin embargo, en el mismo §55, Husserl se pregunta además por el sentido del mundo para los animales. La pregunta por el sentido del mundo para los animales es la pregunta de si existe un mundo para los animales y, al mismo tiempo, cuáles son las formas de dar validez a este mundo. Asumiendo que los animales también dan sentido al mundo, Husserl plantea que este es un mundo humano-animal (cf. Husserl 2008b) en la medida que está revestido por un entrecruzamiento entre significaciones humanas y animales (cf. Husserl 2008a; 2008b; Ferencz-Flatz 2017). En función de esto, seres humanos y animales co-habitan intersubjetivamente un mundo común $\mathrm{y}$, al mismo tiempo, se co-constituyen unos a otros como en el caso de la domesticación y la tenencia de mascotas. En este trabajo quiero mostar el modo en que la domesticación, entendida como este entrecruzamiento entre la vida humana y animal, puede tener consecuencias ontológicas y éticas para la constitución de las subjetividades animales y humanas dependiendo de la fuerza y la riqueza de su convivencia. Mi argumentación sigue tres pasos: en primer lugar, determinaré los pasos fenomenológicos necesarios para dar cuenta de la constitución de sentido de la subjetividad animal y la posibilidad de una constitución de su mundo; en segundo lugar, analizaré un estudio de caso de Tomasello y Call presentado por Leavens (cf. 2014) en el cual dos orangutanes, Chantek y Puti, fueron sometidos a una serie de experimentos para evaluar sus capacidades de comunicación no verbal y cómo éstas fueron aprendidas en contacto con grupos humanos; en tercer lugar, discutiré el fenómeno actual de animales domésticos que alcanzaron un reconocimiento legal en nuestra sociedad, basándome fundamentalmente en la llamada Ley de tenencia responsable de mascotas

y animales de compañía, donde se establecen obligaciones de cuidado de sus mascotas para los dueños de estos animales. Concluiré algunas consecuencias éticas respecto de la domesticación y de cómo el modo de entrecruzamiento entre humanos y animales puede modificar la vida de unos y otros.

\section{Humanos y animales: la co-pertenencia a un mundo}

Para los conocedores de la obra de Husserl no es un misterio que el concepto de mundo es un concepto difícil debido principalmente a que puede ser entendido de 
diversas maneras e, incluso, algunas de ellas pueden parecer confusas ( $c f$. Moran 2012; Carr 1970). Uno de los tratamientos más extensos del concepto de mundo en Husserl es el que se desarrolla en su obra Crisis de las ciencias europeas y la fenomenología trascendental donde, específicamente, éste se entiende como mundo de la vida (Lebenswelt). Para el autor, el mundo de la vida es el mundo humano, el mundo cotidiano en el que vivimos y en el que desarrollamos todas nuestras actividades prácticas y teóricas y que puede ser entendido "en el pleno sentido del mundo para todos [...] el mundo de la cultura" (Husserl 2008a 2281). Esto quiere decir que, en un sentido del mundo, este aparece con significaciones humanas, en relación con comunicades primitivas a partir de la tradición, así como con algunas que nos son territorialmente lejanas o extrañas e, incluso, otras más complejas. No obstante, es claro que este nivel de constitución es, digamos, "superior" y que en el análisis abstractivo podemos retroceder a ámbitos de constitución previos, los cuales metodológicamente nos conducen desde el mundo experimentado cotidianamente hacia estratos de constitución más básicos. Por ejemplo, a través de una abstracción, accedemos al mundo de la experiencia natural, del mundo en tanto naturaleza (Welt-Natur). Este ámbito es descrito como un mundo de cosas (Dingwelt) sin sus predicados espirituales humanos $\mathrm{y}$, entonces, nos encontramos con objetos que son modificaciones de nuestras experiencias cotidianas y que no pueden ser dados sino en forma abstractiva (Husserl 2005). Ejemplo de ello es que cuando bebo agua en un vaso, lo que experimento es fenomenológicamente distinto que lo que experimento cuando investigo la composición química del agua y la denomino como $\mathrm{H}_{2} \mathrm{O}$. Es decir, la posibilidad práctica que me ofrece el agua para beberla, es un significado mundo-vital que no está (necesariamente) presente en la denominación científica del agua, entendida como un compuesto químico de dos átomos de hidrógeno y uno de oxígeno. En un caso estamos ante un objeto de nuestra experiencia directa; en el otro, estamos ante una experiencia de segundo orden ${ }^{2}$. Es como si en el mundo de la vida hubiera siempre un superávit de sentido. De tal modo, avanzando en el examen reflexivo, puedo ir desvistiendo el mundo de la vida de sus predicados humanos e ir determinando estructuras que pertenecen a ámbitos de la constitución previos y determinar sus significaciones correspondientes.

Junto con la experiencia que tengo de cosas relativas a la mera naturaleza, me encuentro también en el mundo con las cosas vivientes (Lebewelt), las cuales pueden ser otros seres humanos y sus producciones culturales, pero también animales de diversos tipos (Moran 2012 195). Según Husserl: “Entre las cosas sin-

1 Ver también Meditaciones Cartesianas $§ 58$.

2 Básicamente, a partir de este hecho se puede establecer la primacía fenomenológica del mundo de la vida sobre toda objetivación científica o de cualquier tipo. 
vida se destacan las humanizadas, cosas que tienen significado a partir del ser humano (por ejemplo, sentido cultural), además, en modo modificado [in abgewandelter Weise], las cosas que remiten con sentido al existente animal" (Husserl 2008a 265). Esto quiere decir que el mundo humano aparece relativo a objetos que, pese a su materialidad, esconden significaciones espirituales, tales como los libros que tengo a mi lado cuando escribo este trabajo, la impresora que está sobre la mesa, el cuadro del día de mi matrimonio con Romina. Todas esas cosas humanizadas adquieren su significado debido a que sobre su base material se establece una capa espiritual que es fundamento de su significatividad. Sin embargo, en conjunto con esas cosas, aparecen unas que podemos llamar animalizadas: miro hacia un lado y veo la cama de mis perros; bajo a buscar un vaso con agua y veo su bebedero. Uno de mis perros, con su patita, lo mueve para que lo llene, pues está vacío y él está sediento. De pronto, sin darme cuenta, piso el pato de hule de mis mascotas y este suena. Todos esos objetos tienen un significado humano en cuanto aparecen en el contexto de mi casa. Sin embargo, todos ellos poseen significados animales entrecruzados con los significados humanos, por cuanto son los objetos de uso de mis perros, poseen significaciones para sus vidas en el encuentro cotidiano que establecen con ellos. Todo esto da cuenta de un mundo en el cual las significaciones humanas y animales se entremezclan para dar paso a una conjunción intersubjetiva.

La posibilidad de establecer esta conjunción intersubjetiva se sostiene, según Husserl, por el hecho de que en un nivel de constitución previo, las subjetividades se co-constituyen en un proceso de comunización (Vergemeinschaftung) a partir de cual, en un nivel más elevado de la constitución, constituyen un mundo intersubjetivo, como un mundo co-constituído por seres humanos y animales. Esto revela que, en función de esas capacidades constitutivas, seres humanos y animales son para Husserl subjetividades trascendentales o constituyentes ( $c f$. Husserl 1973a, 1979; San Martín 2007)³. Sin embargo, en otro nivel del análisis y a pesar que las subjetividades se constituyan desde un primer momento como comunitarias, la fenomenología busca esclarecer el sentido de la alteridad a partir del sujeto que reflexiona. En términos de la Quinta Meditación Cartesiana, la descripción del fenómeno desde el cual podemos obtener el sentido de la alteridad se funda en el análisis fenomenológico de la constitución primaria que se obtiene en el ámbito reducido de la esfera de mi propiedad (Eigenssphäre) (cf. Husserl 1979), nivel en el cual se puede dar cuenta que, fenomenológicamente hablando, el otro se me da desde el comienzo como anímicamente inaccesible (cf. Staehler 2008).

\footnotetext{
${ }^{3}$ El fenómeno de la comunización también puede ser hallado en la dimensión pasiva de la subjetividad. En la generatividad de la constitución pre-yoica, existe un proceso intersubjetivo en el cual la comunización se desenlaza como paridad sexual. Cf. Cabrera, 2013.
} 
Esto significa que la vida anímica del otro solamente se me co-da, se me apresenta y es fenomenológicamente imposible acceder directamente a la vida subjetiva del otro. Lo único que me aparece directamente a la vista es la corporalidad del otro. Esta es, a juicio de Staehler, una de las enseñanzas fundamentales de la Quinta Meditación Cartesiana 4 .

Los animales en cuanto se me presentan como una alteridad lejana, menos comprensible, también señalan el modo de inaccessibilidad en su vida anímica, aunque directamente a través de sus cuerpos. $Y$, pese a que los animales se me dan de manera inmediata en la experiencia como sujetos, en el ámbito de la reflexión fenomenológica puedo determinar modos a partir de los cuales se constituye su sentido de alteridad. Junto con presentarse en el modo de la inaccesibilidad, el animal se da como una modificación de mi subjetividad (cf. Ciocan 2017). En este sentido, en el ámbito de la reflexión que se desliza sobre lo ya constituido, podemos determinar una estructura de modificación (Abwandlung) sobre la que se asienta la comprensión empática que tengo sobre el animal. Como señala la Quinta Meditación Cartesiana, toda experiencia del otro se funda en la empatía (Einfühlung) en la cual la alteridad animal podría entenderse, grosso modo, de dos maneras: a) como una modificación de mi corporalidad; b) como una modificación de las capacidades y estructuras cognitivas que tengo como subjetividad. Esto quiere decir, en primer lugar, que el cuerpo del otro animal me aparece como una "variación anormal o modificación del ser humano" (Ciocan 2017 176), debido a que toda extrapolación empática de sentido me presupone como la norma $\mathrm{y}$, de esta manera, todo lo que me es diferente aparece inmediatamente como anormal. Esto es lo que Husserl quiere decir cuando señala que el animal es anormal (Anomal), pues es una variación de mi humanidad (1979 196). En segundo lugar, el animal me aparece inmediatamente con capacidades cognitivas diferentes. Por ejemplo, el animal me aparece sin las capas racionalidad o pensamiento teórico (San Martín 2007 49, 50; Osswald 2012 592). Además, el animal carece del sentido personalidad (San Martín 2007 56). Otra vez, el animal inmediatamente se me da como una modificación mía, en cuanto que mi subjetividad ofrece la "apercepción originaria y da la norma necesaria" (Husserl 1973a 126), a partir de lo cual concluyo por experiencia directa con la vida animal que esta carece de las capas racionalidad y personalidad. La experiencia del animal en tanto que otro se inserta desde el inicio en mi comprensión de su subjetividad a partir de la empatía y, de entrada, se me manifiesta como siendo un sujeto distinto a mí, un sujeto anormal en el sentido de que la normalidad esta dada por mi mismo como portador de la

\footnotetext{
${ }^{4}$ Y no, como han sostenido algunos, la dificultad de acceder a una dimensión intersubjetiva desde el sujeto, quedando la fenomenología relegada a un solipsismo trascendental.
} 
norma ${ }^{5}$ y que, por consiguiente, el análisis fenomenlógico de la alteridad del animal se ubica metodológicamente en la teoría general de la normalidad.

El mundo que habitamos se co-constituye por la conjunción y el encuentro de subjetividades humanas y animales. Sin embargo, la pregunta por el sentido del mundo del animal es una pregunta que no tiene que ver específicamente con el modo como accedo al sentido de su alteridad, sino más bien al hecho de que el animal posee capacidades constitutivas de un mundo, por cuanto hablamos del mundo del perro, del gato o de la abeja. Además de esto, puedo constatar que el animal me aparece en el mundo, en cuanto percibo su presencia en mi entorno como ejerciendo ciertos poderes constitucionales: "El mundo es un mundo humanizado y animalizado [...] Es un mundo de cultura. Los objetos de este mundo se presentan a sí mismo en la experiencia concreta como armas, casas, objetos de uso (Zweckobjekte) de todos los tipos, como huellas en la hierba [...] Pero es lo mismo con los animales. Al ver 'huellas de animales' podemos 'intuir' que hubo animales presentes y de qué tipo eran" (Husserl 2008b 510). Junto con los objetos del mundo de la vida, en el mundo me aparecen sentidos que remiten a acciones de los animales, como las huellas animales (Tierspuren) que indican su presencia. Esto da cuenta que el mundo no es solamente un mundo cultural, un mundo de la vida, sino que además se entrecruza con acciones o intereses prácticos animales. Cuando trepo un árbol en medio del bosque puedo encontrar nidos vacíos (o habitados) que indican la presencia de animales en el lugar. Caminando por la calle, puedo encontrar bolsas de basura rasgadas y con su contenido desparramado, lo que me indica la presencia de un animal que realizó tal travesura. Entrando yo en mi casa, puedo encontrar el pollo de hule decapitado y, unos pasos más allá, la cabeza del pollo desfigurada por colmillos caninos. Así, asumo que Schnitzel (mi mascota) ha sumado un ejemplar más a la colección de pollos decapitados. Estos ejemplos dan cuenta de presencias animales en esos lugares y, al mismo tiempo, nos permiten anticipar qué tipo de animales eran, como señala la cita de Husserl. En todos esos casos las cosas se me presentan como alteradas por otro tipo de subjetividades, los animales, los cuales, al igual que los seres humanos, modifican su entorno a partir de acciones que son de entrada significativas, que las comprendemos empáticamente como orientadas hacia fines prácticos, como comer o hurguetear la basura o jugar y/o romper objetos.

\footnotetext{
${ }^{5}$ Esto significa que la investigación husserliana de la experiencia animal se enmarca dentro de la teoría general de la normalidad. Sin embargo, es necesario entender que se trata de una cuestión técnica y no de una cuestión ontológica. Los animales, como ha mostrado Depraz (1995), poseen su propia forma de normalidad. Por lo tanto, entender al animal como anormal es una cuestión metodológica que se explica por la necesidad de retrotraernos a la esfera de la propiedad para dar cuenta de la formación del sentido de la alteridad animal.
} 
Los animales que aparecen en el mundo pueden ser de distintos tipos: estos pueden ser animales salvajes (Tiere), animales familiares (Heimtiere) y animales domésticos (Haustiere). Los primeros se presentan como animales de un mundo ajeno (Fremdwelt), en el sentido que no nos son familiares y los encontramos en ecosistemas distintos al que comúnente habitamos. A diferencia de los hombres que habitan estos mundos ajenos ${ }^{6}$, estos animales viven de maneras que son difíciles de comprender para nosotros, debido a que no tenemos una experiencia concreta de sus vidas. Los segundos son aquellos que pertenecen a nuestro mundo familiar (Heimwelt), en el sentido que, pese a que no habitan en nuestras casas, pertenecen al mundo circundante (Umwelt) en el cual vivimos. Los terceros son los animales domésticos, los cuales viven en nuestros hogares y con quienes compartimos una forma de entrecruzamiento directo, en cuanto que tanto podemos comprendernos y generar sentidos compartidos. Por ejemplo, Husserl plantea que estos animales domésticos "en la medida que han sido criados y educados por humanos, han adquirido ciertos rasgos de humanidad" (Husserl 1973b 626). Por ejemplo, los animales domésticos, como gatos y perros, adquieren ciertos rasgos de comportamiento normativo, como orinar en ciertos lugares y no en otros, los cuales son aprendidos por ellos a partir de este entrecruzamiento con seres humanos. En este sentido, domesticar se refiere al proceso a partir del cual los animales son introducidos en nuestro mundo de la vida para de este modo adquirir algunos de nuestros significados y capacidades humanas. Es un proceso de humanización que, sin embargo, no es unidireccional. Mientras que los animales domésticos son sometidos a este proceso de humanización, a su vez, los seres humanos también pueden ser modificados por los animales, en cuanto ellos se transforman en compañeros de vida, dejan sus huellas en nuestros hogares y adecuamos nuestras vidas en función de sus necesidades (cf. Ferencz-Flatz 2017).

Los animales no sólo son parte de nuestro mundo, sino que además forman parte de él, enriqueciendo nuestra experiencia y, por tanto, siendo compañeros subjetivo-constutitivos del mundo en el cual habitamos. Es por esto que tanto seres humanos como animales son considerados por Husserl subjetividades trascendentales. Los animales son seres intersubjetivos porque comparten con nosotros el mundo, nos familiarizamos mutuamente, los incluimos en nuestras actividades y hasta llegan a comprender algunos de nuestros sentidos humanos. Además, los animales exhiben capacidades las cuales podríamos pensar que son específicamente humanas, tales como el lenguaje no verbal. En la segunda sección, presentaré el caso de Chantek y Puti, dos orangutanes que aprendieron la

\footnotetext{
${ }_{6}$ Pese a las diferencias que se establecen entre el modo de vida humana en cada mundo de la vida, existen modos de comprensión mutua que se establecen a partir de la experiencia de un mundo que se comporta de manera concordante. Cf. $\$ 36$ de Crisis.
} 
capacidad de apuntar de manera diferente ( $c f$. Leavens 2014) debido a la manera en que tuvo lugar el proceso de domesticación en cada uno de ellos. Mientras que en el caso de Puti su experiencia no fue enriquecida por un intercambio fluído con seres humanos, en el caso de Chantek su relación con humanos fue clave a la hora de desarrollar capacidades complejas. Este fenómeno podría indicar que la fuerza de entrecruzamiento entre humanos y animales es fundamental en vistas de posibilitar el aprendizaje de características humanas en los animales.

\section{Diferencias en la co-pertenencia al mundo. El caso de Chantek y Puti}

En 1994, Josep Call y Michael Tomasello de la Universidad Emory, realizaron una serie de experimentos sobre la capacidad de apuntar en dos orangutanes, Chantek y Puti. Chantek nació en cautiverio en 1977 y desde los nueve meses de edad fue introducido en un programa de entrenamiento lingüístico en la Universidad de Tennessee. Durante su formación en este ambiente, no sólo aprendió la utilización de signos, sino que también algunas de las reglas de comportamiento e interacción propiamente humanas. Este proceso de entrenamiento y asimilación de características humanas ha sido llamado enculturación por los especialistas (Leavens 2014 84). Puti fue un orangután hembra que nació y creció en un centro de investigaciones biomédicas llamado Yerkes Primate Center. La orangután fue criada hasta los dos años por una madre orangután sin capacidades maternas, teniendo después contacto sólo con congéneres de su misma edad. Tal modo de crianza socialmente empobrecida fue llamada por algunos científicos crianza por humanos (Leavens 2014 84). Las diferentes formas de entrecruzamiento de Chantek y Puti con otros seres humanos se hicieron patentes en los resultados de los experimentos liderados por Call y Tomasello. Por ejemplo, los investigadores notaron que, al comienzo, sólo Chantek apuntaba ${ }^{7}$, mientras que Puti era incapaz de hacerlo. Por ese motivo, tuvieron que someterla a un entrenamiento paralelo. Finalmente, sólo consiguieron que apuntara con toda la mano, a diferencia de Chantek quien podía apuntar con el dedo índice.

Call y Tomasello realizaron un primer experimento en el cual los orangutanes tenían que escoger entre tres contenedores, uno de ellos con comida visible, y apuntar en cada caso, ya sea al contenedor que mostraba la comida directamente (condición sin herramienta) o a la herramienta que permitía acercar el contenedor con comida que estaba cubierto por una malla de alambre (condición con herramienta). En este primer experimento, tanto Chantek como Puti apuntaron

\footnotetext{
7 Hay otros casos similares. Por ejemplo, en un artículo de la revista online Aeon, Leavens cuenta que en 1994 conoció a un chimpancé llamado Clint en la Georgia State University que tenía la capacidad de apuntar, pero que no se sabía muy bien cómo lo había aprendido. Ver Leavens 2019.
} 
con exactitud (Leavens 2014 85). Sin embargo, se observó una significativa diferencia en el segundo experimento. Se trataba de un test más difícil, en el cual se mantenían los tres contenedores (uno de ellos con comida), pero se le agregaba una dificultad. En este caso, la herramienta era ocultada por uno de los experimentadores detrás de una de las tres mantas que colgaban de una pared en frente de las jaulas de los orangutanes. La tarea que debían realizar Chantek y Puti era: a) ubicar y apuntar hacia la herramienta oculta y b) ubicar y apuntar al contenedor con comida. De veintidós ensayos, Chantek inicialmente cometió algunos errores, pero de los últimos diez ensayos, nueve de ellos los respondió acertadamente. Puti no respondió ninguno de los veintidós ensayos de manera correcta cuando se trataba de la herramienta escondida.

En el tercer experimento, se volvió a utilizar los tres contenedores, pero no las herramientas. En este caso, se ponía comida de manera que los orangutanes no pudieran ver cuál de los contenedores era llenado. Luego, se destapaban y acercaban a las jaulas de los animales $y$, antes de abandonar el lugar, el experimentador apuntaba hacia el contenedor que había sido llenado con comida. Después de eso, llegaba otro experimentador que hacía que los orangutanes apuntaran al contenedor lleno de comida, sólo dándosela cuando ambos orangutanes apuntaban al contenedor correcto. En este caso, Chantek nuevamente superó a Puti, logrando correctamente llegar más arriba de la media de efectividad, mientras que Puti sólo acertó en un 33\% de los intentos (Leavens 2014 86). Call y Tomasello concluyeron después de esta serie de experimentos que la experiencia previa de los animales es importante, especialmente aquella que está vinculada con sus relaciones con seres humanos (Leavens 2014 87). Este mismo modelo de experimentos fue realizado por los investigadores dos años después, pero esta vez la muestra incluía además niños de dos años y medio junto con un grupo de orangutanes, en el cual estaba Chantek. Los resultados arrojaron que el $70 \%$ de los niños pudo identificar correctamente la información ofrecida por las señas de los experimentadores. En el caso del grupo de orangutanes, sólo Chantek respondió correctamente la prueba.

Leavens señala que, a partir del análisis de esta serie de experimentos, podemos extraer tres importantes conclusiones. En primer lugar, estos experimentos muestran que el proceso de enculturación previo a la experimentación con animales es relevante, porque muestra que la lectura de señas por parte de los orangutanes (que también se aplica en chimpancés) es una posibilidad que surge a partir de que ellos sean expuestos a "las mismas oportunidades de entrar en relación con humanos que las que les brindamos a nuestros niños humanos" (Leavens 2014 92). En segundo lugar, dada la diferencia en el modo de enculturación que podemos establecer en los dos orangutanes, se 
concluye que los grupos muestrales son completamente diferentes y que no podemos mezclar animales enculturados (al modo de Chantek) con animales que sólo han sido criados al interior de ambientes humanos, pero que no se les ha brindado la oportunidad de generar ricas interrelaciones con seres humanos. En tercer lugar, los experimentos permiten concluir que tanto niños humanos como orangutanes enculturados responden de manera similar a los test de habilidades sociocognitivas, como la respuesta ante el lenguaje de señas. Estas tres conclusiones son importantes, pues ilustran a los investigadores sobre la necesidad de considerar los procesos de enculturación previos de los sujetos de estudio, muestran que esta diferencia marca una fundamental distinción en la respuesta esperada de esos sujetos y permiten rechazar el supuesto comúnmente aceptado por la comunidad científica de que el éxito que tienen los niños por sobre los animales que son sometidos a estos experimentos radica en una diferencia de las capacidades cognitivas que exhiben ambos grupos de sujetos. De tal modo, se puede decir que la brecha entre la cognción social animal y la cognición social humana en la comprensión del lenguaje no verbal depende, en alguna medida, del modo en que ciertos tipos de animales han sido incorporados a mundos humanos o, más exactamente, del modo como esos animales han sido "criados y educados por humanos", es decir, del modo de domesticación a la cual han sido sometidos.

En la tercera sección de este trabajo, mostraré algunas consideraciones éticas que se pueden extraer acerca de la domesticación basándonos en la evidencia fenomenológica y científica de las subjetividades animales anteriormente presentada.

\section{Chantek y Puti como otros yo. Algunas consideraciones éticas de la domesticación}

La fenomenología de Husserl nos ofrece una consideración del modo en que la vida animal se inserta constitutivamente en nuestra experiencia del mundo. Nuestro mundo no está constituido únicamente por seres humanos, sino que los animales, en tanto subjetividades trascendentales, poseen poderes constitutivos de sus propios mundos circundantes $\mathrm{y}$, en alguna medida, de los nuestros. Es por eso que el mundo de la vida es, a juicio de Husserl, un mundo humanizado y animalizado: los animales son sujetos co-constitutivos del mundo en que vivimos. En el caso de Chantek y Puti, nos encontramos con dos sujetos en los cuales el proceso de enculturación o domesticación ha tenido lugar de maneras diferentes. Con Chantek tenemos un claro ejemplo de un animal que ha sido humanizado, pues no sólo ha aprendido a comunicarse con humanos sino que, como plantea su cuidadora en una conferencia dada en 2014, el orangután también ha 
manufacturado artesanías y ha mostrado conductas empáticas (cf. Tedx 2014) ${ }^{8}$. En el caso de Puti, su inclusión en el mundo humano ha sido más bien a partir de la despreocupación, de una inclusión empobrecida de interrelaciones que incluyan al animal en el mundo de la vida humano de manera compleja y constante, como pareciese ser el caso de Chantek.

A partir de lo anterior, y teniendo en cuenta que los animales están insertos en distintos modos de pertenencia a nuestro mundo de la vida, ya sea en el modo de sujetos experimentales o de mascotas, pero también como animales salvajes, establecemos relaciones interpersonales con ellos de modo similar a como las establecemos con nuestros familiares y amigos, así como también con desconocidos. En el caso de los animales domésticos es interesante señalar que ellos han sido los primeros en obtener reconocimiento legal en las sociedades actuales ( $c f$. Ferencz-Flatz 2017). En Chile, actualmente los dueños de mascotas tienen obligaciones legales con ellas ${ }^{9}$, tales como inscribirlas en la municipalidad donde habitan, implantarles un microchip y responder ante los daños que puedan causar. Sin embargo, algunas de las obligaciones más interesantes que contempla esta ley en relación con el tema de este trabajo, son las que establecen que los dueños tienen ciertos compromisos éticos con su mascota o animal de compañía, tales como "proporcionarle alimento, albergue y buen trato, brindarle los cuidados veterinarios indispensables para su bienestar y no someterlo a sufrimientos a lo largo de su vida" (cf. Ley №21.020 2017). Este punto demuestra que los entrecruzamientos entre animales y humanos no se desenvuelven solamente en forma paralela, es decir, el animal no constituye su mundo de forma paralela a como nosotros constituimos el nuestro, sino que da cuenta de una interferencia entre unos y otros, refiere un afectarse mutuamente que hace que el mundo humano sea a su vez un mundo animal (el caso de la Ley Cholito) y que un mundo animal sea a su vez un mundo humano (el caso de Chantek). Nuestras mascotas modifican el mundo familiar en el cual habitamos, incluso tomando el control de nuestras vidas. Al mismo tiempo, nos acompañan y son parte fundamental del día a día. Para ellos, nosotros establecemos pautas normativas de comportamiento, de modo que, como señala Husserl, adquieren ciertos rasgos de humanidad. Sin embargo, esta normatividad no es unidireccional, debido a que las mascotas nos obligan a repensar nuestras obligaciones legales en el trato diario que establecemos con ellas y, de ese modo, se entrelaza su modo de vida con el nuestro.

\footnotetext{
8 Claramente, esto suscita la pregunta de hasta qué punto es ético el proceso mismo de la domesticación. No obstante, esta pregunta merece su propia investigación.

${ }_{9}$ A través de la ley 21.020 la llamada "Ley de Tenencia Responsable de Mascotas y Animales de Compañía", popularmente llamada "Ley Cholito".
} 
Para finalizar, quiero señalar que es evidente que existen otros modos de entrecruzamiento entre el ser humano y el animal que no necesariamente corresponden a la domesticación. Tales casos podemos encontrarlos en los zoológicos, los mataderos, e incluso en la referencia a animales humanizados que nos muestran los programas de televisión de dibujos animados (Ferencz-Flatz 2017 229). Es evidente que cada forma de relación, ya sea la humanización que se da en la domesticación o la pérdida del respeto por la vida que se da en casos de explotación animal, conlleva intrínsecamente nuevas y quizá más radicales consecuencias éticas para la vida planetaria. Esas investigaciones son necesarias en medio de la toma de conciencia juvenil del problema ecológico, como en el caso de Greta Thunberg, o incluso en las tradiciones ancestrales de algunos de pueblos indígenas del mundo que aún subsisten; no obstante, esas necesarias temáticas deben ser abordadas en otras investigaciones. En conclusión, pese a que las subjetividades animales carezcan de algunos estratos constitutivos de la subjetividad trascendental humana, pueden adquirir algunas de estas capacidades en su entrecruzamiento con seres humanos. Esto dependerá de la fuerza y la riqueza del entrecruzamiento entre ambos tipos de subjetividades. Al mismo tiempo, a partir de esta misma relación surgen ciertas consecuencias éticas que regulan la convivencia entre seres humanos y mascotas. En otras palabras, existe una comunidad intersubjetiva entre seres humanos y animales la cual está situada en un mundo común que, si bien se diferencia por ciertos estratos de constitución subjetiva que están ausentes en la vida animal, plantea la pregunta de en qué sentido este mundo común puede ser filosóficamente develado en su constitución humana y animal.

\section{Bibliografía}

Cabrera, Celia. "Intersubjetividad a priori y empatía". Ideas y valores 62 (2012): 7193.

Carr, David. "Husserl's Problematic Concept of the Life-World". American Philosophical Quarterly 7 (1970): 331-339.

Ciocan, Cristian. "Husserl's Phenomenology of Animality and the Paradoxes of Normality". Human Studies 40 (2017): 175-190.

Ferencz-Flatz, Christian. (2017). "Humanizing the Animal, Animalizing the Human: Husserl on Pets". Human Studies 40/2 (2017): 217-232.

Husserl, Edmund. Zur Phänomenologie der Intersubjektivität. Texte aus dem Nachlass. Zweiter Teil. 1921-28. Den Haag: Martinus Nijhoff Publishers, 1973a. 
Husserl, Edmund. Zur Phänomenologie der Intersubjektivität. Texte aus dem Nachlass. Dritter Teil. 1929-35.. Den Haag: Martinus Nijhoff Publishers, $1973 b$.

Husserl, Edmund. Meditaciones cartesianas. España: Ediciones Paulinas, 1979.

Husserl, Edmund. La Crisis de las Ciencias Europeas y la Fenomenología Trascendental. Argentina: Prometeo Libros, 2008a.

Husserl, Edmund. Die Lebenswelt. Auslegungen der vorgegebenen Welt und ihrer Konstitution. Texte aus dem Nachlass (1916-1937). Dordrecht: Springer, $2008 b$.

Leavens, David. "The Plight of the Sense-Making Ape". Enactive Cognition at the Edge of Sense-Making, eds. Massimiliano Cappuccio y Tom Froese. United Kingdom: Palgrave MacMillan, 2014. 61-104

Leavens, David. The pointing ape. Aeon. 1 de octubre de 2019. Web. 20 Oct. 2019.

N.P. Ley No 21.020. Biblioteca del Congreso Nacional. 2 de agosto de 2017. Web. 21 Ago. 2019.

Moran, Dermot. Husserl's Crisis of the European Sciences and Transcendental Phenomenology, An Introduction. England: Cambridge University Press, 2012.

Osswald, Andrés. "Sobre la subjetividad animal o de la animalidad del sujeto: un recorrido por la reflexión husserliana sobre los animales", Anuario Filosófico 43/3 (2012): 589-614.

San Martín, Javier. "La subjetividad trascendental animal". Para una filosofía de Europa, ed. Javier San Martín. España: Biblioteca Nueva, 2008. 39-67.

Staehler, Tanja. "What is the Question to Which Husserl's Fifth Cartesian Meditation is the Answer?", Husserl Studies, 24 (2008): 99-117.

Tedx, Talks. Chantek, the first orangutan person. Lyn Miles, TEDxUTChattanooga. Youtube. 11 Nov. 2014. Web. 20 Oct. 2019. 